\title{
APLICACIÓN DEL COSTEO BASADO EN ACTIVIDADES A LAS PEQUEÑAS Y MEDIANAS EMPRESAS DEL SECTOR INDUSTRIAL DE LA CIUDAD DE CARTAGENA
}

\author{
JESUS BLANQUICETT TORRALVO *
}

\section{RESUMEN}

El propósito del siguiente artículo es presentar una aplicación metodológica del costeo basado en actividades a las pequeñas y medianas empresas del sector industrial de la ciudad de Cartagena, como resultado de un trabajo de investigación aplicado a una muestra de estas empresas y consistente en el diagnóstico en el uso de un sistema de costeo por actividades dentro de las organizaciones consultadas. Para lo anterior, se estudiará y analizará la evolución del sistema de costos moderno en sus cuatro Fases según Kaplan y Cooper, adicionalmente, se realizará recolección de información primaria cómo; observación directa, aplicación de instrumentos y entrevistas para establecer la Fase de Costos en que se encuentra la empresa analizada y sus implicaciones en la toma de decisiones. El hallazgo de mayor significancia del estudio realizado se centra fundamentalmente en que pudo corroborarse la existencia de subsidio cruzado entre productos que se presenta en el costeo tradicional, como por ejemplo, la leche líquida pasterizada donde el tiempo de proceso es mayor en la medida que se reduce el tamaño de la bolsa y sin embargo el costeo tradicional calcula para todos una tasa de CIF equivalente.

Palabras clave: Costeo basado en actividades, Inductor de costos, actividades primarias, centro de actividades, actividades de apoyo, centro de costos, parámetros de consumo, recursos.

Clasificación JEL: D20, D21, D24, L23.

\footnotetext{
* Programa de Contaduría Pública de la Facultad de Ciencias Económicas - Universidad de Cartagena. Correo Electrónico: jesusblanquicett@yahoo.es
} 


\begin{abstract}
The globalización of the economy, the dynamic technological changes, and the integration of the markets, have taken to the companies to establish strategies that allow them to compete with quality and productivity, of such form that their products have acceptance in the market. Colombia is not other people's to the influence of those factors, agravated with the problems in economic, political and social matter. Those circumstances are economically affecting the companies of the productive sector, between these, to the small and medium companies of the industrial sector of the city of Cartagena. Within this context a good and effective system of costs would take to the companies to compete with prices, since he is one of the most important factors, along with the quality and the good service. Nevertheless the present systems of costs have a very old seal. From which the importance of this work is based on the application of the verified theoretical concepts and widely spread of Activity Based Costing, with the purpose of giving answer to the new exigencies that the surroundings are requiring to him to this type of companies in its sector. Finally the general conclusions and some recommendations appear.
\end{abstract}

Key words: Activity Based Costing, costs drivers, primary activities, center of activities, activities of support, center of costs, parameters of consumption, resources.

JEL Classification: D20, D21, D24, L23.

\title{
INTRODUCCIÓN
}

La globalización de la economía, los dinámicos cambios tecnológicos, y la integración de los mercados, han llevado a las empresas a establecer estrategias que les permitan competir con calidad y productividad, de tal forma que sus productos tengan aceptación en el mercado. Colombia no es ajena a la influencia de esos factores, agravado con los problemas en materia económica, política y social que se han agudizado últimamente en el país. Esas circunstancias están afectando económicamente a las empresas del sector productivo, entre estas, a las pequeñas y medianas empresas del sector industrial de la ciudad de Cartagena.

Dentro de este contexto un buen y efectivo sistema de costos llevaría a las empresas a competir con precios, ya que es uno de los factores más importantes, junto con la calidad y el buen servicio. Sin embargo los ac- 
tuales sistemas de costos tienen un sello muy antiguo, los cuales se fueron elaborando progresivamente entre 1850 y 1910, en particular en la gran industria norteamericana.

Las necesidades de información y el entorno han cambiado considerablemente, por lo que, los sistemas de costos tradicionales ya no cumplen con los objetivos para los que fueron diseñados, son insuficientes, y a veces equivocados, para suministrar información acorde con las necesidades empresariales actuales. De allí que la importancia de esta investigación se fundamente en la aplicación de los conceptos teóricos comprobados y ampliamente difundidos del Costeo Basado en Actividades, con el fin de darle respuesta a las nuevas exigencias que el entorno le está requiriendo a este tipo de empresas en su sector.

En este trabajo se realiza, en primer lugar, unas encuestas teniendo en cuenta dos formularios: el primero dirigido al responsable de los costos y el segundo dirigido al gerente o administrador de la empresa, donde el objetivo principal de la primera encuesta fue determinar en qué fase de costos se encuentran las empresas del sector industrial de la ciudad de Cartagena, y la segunda conocer de primera mano la utilización y la importancia de los costos para la gestión administrativa. En segundo lugar, de acuerdo con los resultados obtenidos en las encuestas, se procedió a elegir la empresa para la aplicación del costeo basado en actividades. En este sentido se escogió una empresa de alimentos (de productos lácteos), donde las técnicas de recolección de la información se fundamentaron sobre el análisis documental, la entrevista no estructurada, la observación directa de los procesos de la empresa, la cual implicó identificar las actividades, tomar los tiempos de máquina y hombre y establecer las otras bases de distribución de costos indirectos de fabricación e inductores de costos. La información recolectada se organizó, analizó y procesó de tal forma que permitió la aplicación del costeo basado en actividades en dicha empresa.

\section{REFERENTES TEÓRICOS}

\section{Desarrollo Y Evolución De Los Costos}

El desempeño de una empresa ha venido midiéndose en la práctica a través de sus estados financieros, siendo el más utilizado por los gerentes el estado de resultados, que es donde se aprecia el impacto inmediato de la contabilidad de costos, el cual es más complejo de analizar cuando se fabrican varios productos. A continuación se realiza un breve recorrido por la historia para comprender el estado actual y las consideraciones que se tienen hoy, de la contabilidad de costos. 
El desarrollo y evolución de los costos ha sido paralelo al desarrollo de la producción y los avances tecnológicos. Las primeras manifestaciones de la producción aparecen en forma artesanal, la agricultura, al principio, era desarrollada por cada comunidad utilizando, en primera instancia el esfuerzo humano, posteriormente por la domesticación se utilizaron los primeros animales de carga. En esa época el concepto de costo no era tenido en cuenta y mucho menos su importancia.

El paso del autoconsumo familiar a una economía de cambio, permitió la aparición de la oferta y por lo tanto el establecimiento regular de productos para su Intercambio, la identificación de esta práctica dio origen paulatinamente a la formación de federaciones de tribus que con base en un sólido concepto de propiedad, fue el origen de mercados permanentes y por tanto la aparición de algunas actividades contables (1).

Fue así como del siglo XI al XIV en Europa, la contabilidad se fue perfeccionando con motivo de las nuevas necesidades del creciente intercambio comercial y crediticio, dando como resultado la aparición de la contabilidad financiera por partida doble, tal como la conocemos actualmente. En esta época las actividades de transformación no eran relevantes, por lo que la contabilidad de costos no era imprescindible.

Un hecho importante que se resalta es que en 1426, a medida que el comercio y el poder marítimo de Venecia crecieron, sus ciudadanos contemplaron la necesidad de una flota armada para proteger su comercio, poniendo en operación su propio astillero gubernamental, el Arsenal. La contabilidad en este astillero fue tan importante como en los negocios. A mediados del siglo XV se contrataron específicamente tenedores de libros. En un intento hacia la eficiencia, el astillero llevó una cuenta estricta de monedas, materiales y hombres. También se utilizó una forma temprana de contabilidad de costos (2).

El periodo de la primera revolución industrial que va de desde finales del Siglo XVIII, hasta la primera mitad del Siglo XIX, se caracterizó por grandes transformaciones económicas y sociales, relacionadas con las invenciones mecánicas, el origen del Capitalismo y de la Empresa como unidad productiva. En este período de grandes cambios, la contabilidad de costos toma un carácter muy importante, para satisfacer la demanda de información que los empresarios estaban requiriendo.

La revolución industrial inició la concepción de las fábricas de producción masiva, se crearon grandes empresas, tanto comerciales como indus- 
triales. Luego debido al desarrollo tecnológico que facilitó la realización de los procesos productivos, se vio claramente la existencia del otro elemento representativo del costo, por su incremento sustancial y que no era incluido en la determinación del costo total del producto, permitiendo el inicio de una nueva fase para los métodos de costeo y que dio origen al tercer elemento del costo, conocido de manera generalizada, como Costos Indirectos de Fabricación (CIF).

Se podría decir entonces, que la contabilidad de costos tiene un origen muy antiguo, a mediados del siglo XV. Que entre el Siglo XVIII y el siglo XIX, la determinación del costo del producto se hizo un poco compleja, como resultado del crecimiento de las empresas, los procesos, la producción y por tanto los costos, los cuales incluían, los materiales directos, la mano de obra directa y los costos indirectos de fabricación. Sin embargo en esta época los CIF se asignaban de acuerdo con una sola base de distribución (Volumen de producción, Horas Hombres u Horas Máquina).

Es así como entre los años de 1889 a 1895, aparece el control de gestión industrial, a través del cronometraje de los tiempos, el seguimiento de la mano de obra directa, los estándares y la asignación de los costos indirectos mediante los tiempos de mano de obra o de los tiempos de máquina, dando como resultado el desarrollo de la contabilidad analítica (3).

En esta época, las empresas que fabricaban productos distintos y personalizados como herramientas para maquinaria, tenían que conocer los costos de los recursos utilizados para producir cada artículo. Esta información era necesaria para determinar la rentabilidad de las diferentes líneas de producto y para ayudar a determinar los precios que se pondrían, especialmente a los productos personalizados, a los presuntos clientes.

Los ingenieros interesados en la gestión científica, como Frederick Taylor, Fayol, Emerson, Church y Towne, desarrollaron procedimientos para medir, con considerable detalle, la cantidad de materiales, mano de obra y de tiempos de máquina necesarios para fabricar productos individuales. Esta información, era recogida, primordialmente, para mejorar y controlar la eficiencia de las operaciones de producción. Las técnicas de cálculo de costos desarrolladas en ésa época, proporcionaron las bases para los sistemas de costos estándar, que siguen siendo utilizados en la actualidad por muchas organizaciones (4).

El periodo de 1930 a 1980 se ha denominado como la "Era del Conocimiento", debido al desarrollo de la tecnología de la información (Infor- 
mática), que influyó no solo en las industrias, sino en los comercios y los servicios, así como en todas las áreas de la sociedad. Este período se caracterizó por un entorno competitivo relativamente constante y de organización fundamentado en la división del trabajo, la mecanización, las economías de escala, y donde la búsqueda continua de incremento de la productividad era una prioridad. Estas circunstancias le permitieron a la contabilidad de costos un verdadero auge ampliando su campo de actuación, que dio como resultado la aparición de la contabilidad gerencial (utilizar la información contable para la planificación y el control, con el fin de la toma de decisiones directivas) (5).

Se ha observado, de acuerdo con este breve recorrido por la historia que, las empresas industriales en la creación de los métodos de costeo, inicialmente le dieron una mayor importancia a los materiales directos, por lo que se consideraba el factor más representativo en la transformación, luego, a través del análisis de los procesos se determinó la importancia del uso de la mano de obra directa, y por último, debido al avance de la industria y al desarrollo tecnológico, se vio la necesidad de incluir en el costo de producción a los costos indirectos de fabricación, por empezar a ser éste muy representativo.

\section{Costos Tradicionales Y Costos Contemporáneos}

En este apartado, se plantean las diferencias existentes entre las metodologías de distribución del costeo tradicional y el costeo contemporáneo, con el fin de observar y analizar las ventajas y desventajas de cada uno.

Históricamente se han venido utilizando el volumen de producción, las horas hombres o las horas máquina como base de distribución de los costos indirectos de fabricación (llamada también de base unitaria), sin tener en cuenta si dicha base unitaria tiene relación de causalidad con el origen de los CIF. Los costos indirectos de fabricación son asignados a los productos con base en una tasa predeterminada, la cual se obtiene seleccionando un criterio de aplicación que normalmente no contempla la relación causa-efecto de la incursión del producto en dicho costo. A ésta forma de determinar el costo de producción se le denomina en la actualidad, "costeo tradicional".

Es así, que a mediados de la década de 1980, aparece el Sistema Costos Basado en Actividades (Activity Based Costing, ABC) cuyos autores Robert Kaplan y Robin Cooper, realizaron sus primeras investigaciones partiendo de la observación de las prácticas de grandes compañías norteamericanas. Esta forma de distribución de costos (ABC), responde a la necesidad de corregir los procedimientos de asignación tradicionales, basados general- 
mente en el volumen de fabricación o en el tamaño físico de los productos, que con el incremento en el número de artículos diferentes ofertados por la empresa y, con el cambio en la estructura y comportamiento de los costos, han perdido validez, por producir información distorsionada del costo.

Plantean además (Kaplan y Cooper), que el costo de los productos debe comprender los costos de las actividades necesarias para fabricarlo y que son las actividades y no los productos, quienes absorben los costos para llevar a cabo la producción. Convirtiéndose ésta, en una nueva forma de asignación y distribución de costos indirectos y a la cual se le ha dado el nombre de "Costos Contemporáneos" (6). Es por tanto, sustancialmente más objetivo en la asignación de los costos indirectos a los productos que dan origen a los costos.

Lo desarrollado por los autores mencionados, indica que para implementar el costeo basado en actividades, la organización debe realizar los siguientes pasos:

1. Identificar las actividades que realizan sus factores productivos indirectos y de apoyo.

2. Determinar cuánto cuestan los recursos que consume la empresa en cada una de sus actividades, para esto se utilizan los inductores de recursos.

3. Identificar los productos, servicios y clientes u objetos de costos, que son la razón que tiene una organización para realizar actividades.

4. Posteriormente, seleccionar los inductores de costos o cost driver de las actividades que vinculan los costos de las actividades con los productos, los servicios y los clientes.

La forma de correlacionar los recursos, las actividades primarias y secundarias con los objetos de costos, finales y provisionales, se hace a través de los inductores de costos o costs drivers. Estos se clasifican en los siguientes tipos:

- Drivers de Recursos. Sirven para asignar los recursos a las actividades, los procesos o centros de costos. Tales como; área, volumen, horas hombre etc.

- Drivers de actividades. Existen tres tipos de inductores, de acuerdo con la función a la cual se asigna el costo de las actividades al objeto del costo: 
> Por transacción. Tales como el número de tiradas de producción, de unidades producidas o de clientes atendidos.

> Por duración. Tales como tiempo para preparar una tirada, horas de inspección u horas de mano de obra directa. Representa el tiempo requerido para llevar acabo una actividad.

> Por intensidad. Asigna directamente los recursos utilizados cada vez que una actividad es realizada, requiere un sistema por órdenes para rastrear todos los recursos utilizados por una actividad en particular, lo que lo hace más exacto pero también más costoso.

- Drivers Fijos. Son inductores que no cambian en un tiempo determinado, normalmente un año, tales como: el área, el número de empleados y los kilowatios Instalados.

- Drivers Variables. Se refieren a inductores que cambian en la medida que aumentan o disminuyen las actividades, el volumen de producción, los servicios y los clientes.

Robert S. Kaplan y Robin Cooper (1980), sugieren la forma en que la moderna gestión de costos puede y debe ser aplicada tanto en las empresas industriales como a las de servicio. Ilustran sobre el potencial y las posibilidades que ofrecen los sistemas avanzados de $\mathrm{ABC}$.

De acuerdo con las características de los modelos de costos existentes en las empresas hacen énfasis en la existencia de cuatro fases que coexisten, dos de las cuales representan a los sistemas de costeo tradicional y dos a los contemporáneos y que explican la evolución de ésta metodología de costos. Las cuatro fases son las siguientes:

FASE I: La constituyen los sistemas de costeo históricos en los cuales no se hace distinción de costos fijos y variables, ni entre reales y estándares. No se posibilita la planeación ni el control.

FASE II: Se ubican en esta fase los costos estándar y los presupuestos flexibles en un intento de posibilitar la planeación y el control; se hace distinción entre los costos fijos y variables. Son útiles para valorar las existencias para los propósitos de los informes de contabilidad externa.

FASE III: Es la aplicación del costeo basado en actividades tomando como base costos reales; su diferencia con los sistemas de costeo tradicionales de Fases I y II es el reparto de los costos indirectos de fabricación, el cual se realiza en función de las actividades relevantes de la empresa. 
FASE IV: Se aplica igualmente el costeo basado en actividades, pero utilizando el enfoque adicional del cálculo de costeo estándar y análisis de variaciones.

Por último, Douglas T. Hicks (7) plantea una solución metodológica denominada: costeo basado en actividades en minúscula "abc", para las PYMES, como solución al problema que tienen algunas empresas, que por su tamaño se les dificulta contar con un costeo basado en actividades.

En esta metodología las actividades son definidas como aquellos grupos de procesos o procedimientos relacionados entre sí que, en conjunto, satisfacen una determinada necesidad del trabajo de la empresa, donde el centro de actividades de la gran empresa se convierte en la actividad de la empresa pequeña.

Por lo anterior, se destaca que una de las diferencias importantes entre el costeo tradicional y lo que propone el costeo basado en actividades, es que mientras, el primero busca distribuir los costos entre los productos, utilizando de manera subjetiva una tasa predeterminada, el ABC realiza una metodología, utilización de varios inductores de costos, que busca estar lo más ajustado posible con la realidad para asignar los costos a los productos o servicios que una organización tiene para ofrecer a sus clientes.

\section{Situación De Las Pymes Industriales De Cartagena}

En la ciudad de Cartagena de Indias, según información de la Cámara de Comercio, existían en el año 2004 un total 86 PYMES pertenecientes al sector industrial dedicadas a diferentes tipos de negocio, discriminadas de la siguiente manera; $26 \%$ alimentos, 24\% metalmecánicas, $12 \%$ químicas y las demás no pasan del $9 \%$. Por lo tanto en la muestra escogida que fue de 21 empresas se tuvieron en cuenta todos los negocios de acuerdo con su participación en el total del sector.

El tamaño de la muestra para realizar el trabajo de campo fue de $21 \mathrm{em}$ presas, de las cuales 6 son de alimentos, 6 de metalmecánica, 2 de plásticos, 2 de cuero y 1 por cada una de las empresas restantes. Se observa que el 51\% corresponden a las empresas más representativas (alimentos y metalmecánica), guardando relación con la participación en el total de las empresas del sector, que es de 50\% (alimentos 26\% y metalmecánica 24).

Este trabajo de investigación, se realizó teniendo en cuenta dos formularios de encuestas, el primero dirigido al responsable de los costos y el segundo al gerente o administrador de la empresa, donde el objetivo 
principal del primer formulario de encuesta fue determinar en qué fase de costos se encuentran las empresas del sector industrial de la ciudad, y el segundo conocer de primera mano la utilización y la importancia de los costos para la gestión administrativa.

Así pues, los resultados obtenidos se presentan a continuación.

\section{FASE DE COSTOS DE LAS EMPRESAS INDUSTRIALES DE CARTAGENA}

Del total de las empresas encuestadas, el 52.63\% no tienen implantado un sistema de costos, el $47.37 \%$ indicó tener sistema de costo. Este resultado muestra que hay un gran número de empresas del sector industrial que ni siquiera están en Fase (1) de costos (contar con un sistema de costos con base en costos reales). Lo cual es muy preocupante, ya que no cuentan con una herramienta (sistema de costos) que les sirva de base para la toma de decisiones, en este mercado competitivo.

Se pudo establecer, que el $44.44 \%$ de las empresas tienen implantado el sistema de costos por procesos, el $33.33 \%$ el sistema de costos por ordenes de producción y el $22.22 \%$ tienen otra forma de determinar sus costos (cálculos empíricos efectuados por los gerentes). Estos últimos no corresponden a un sistema. También se observa la nula participación del sistema de costos basado en actividades, lo que brinda grandes posibilidades para investigar sobre su implementación.

De las empresas que tienen sistemas de costos, el 55.56\% utilizan las unidades producidas como base para distribuir los costos indirectos de fabricación, el $22.22 \%$ utilizan el costo del material directo, el 11.11\% utilizan las horas maquinas y un $11.11 \%$ utilizan el costo primo (costo de material directo + costo de la mano de obra directa), lo que indica que la base utilizada para la distribución del costo indirecto de fabricación, en estas empresas no corresponde, si se tiene en cuenta que este elemento del costo está conformado por una serie de conceptos de costos tales como; mano de obra indirecta, energía, depreciación, seguros, materiales indirectos, impuestos, gas, agua, repuestos, honorarios, etc., los cuales son generados por diferentes causas. Por lo tanto no es razonable ni objetivo relacionar todos los conceptos con una sola base de distribución.

De las empresas que contestaron no tener sistema de costo, con relación al total, $21.05 \%$ son metalmecánicas, $15.79 \%$ de alimentos, $5.26 \%$ químicas, $5.26 \%$ de litografía y $5.26 \%$ de materiales de construcción, para un to- 
tal de $52.63 \%$ empresas que no tienen sistema de costo. Se observa en este resultado la ausencia de sistema de costos en las empresas de alimentos que es donde se aplicará el costeo basado en actividades.

Con relación a los costos indirectos de fabricación (CIF), se pudo establecer su relación porcentual frente al costo primo (MD Y MOD). Se observa que el $89.47 \%$ del total de las empresas consideraron que los costos indirectos representan del costo primo un porcentaje que está entre $1 \%$ a $20 \%$, Sin embargo el $5.26 \%$ consideraron que el costo indirecto es un porcentaje mayor del $30 \%$, otras empresas que también son el $5.26 \%$ de la muestra, simplemente no respondieron la pregunta. Con este resultado no se podría afirmar si los CIF son relevantes o no, porque una cifra puede ser relativamente insignificante pero absolutamente importante o viceversa. También las dos cifras pueden ser importantes o las dos insignificantes. En el caso de la empresa escogida para la aplicación del costeo basado en actividades, los CIF mensuales ascienden a $\$ 356.264 .605$ y representan el $22 \%$ del costo primo, esta es una cifra importante vista de manera absoluta como de forma relativa.

Hasta ahora, el análisis realizado de acuerdo con los resultados obtenidos en las encuestas, demuestran en gran medida la inexistencia de sistema de costos en un gran número de empresas del sector industrial de la ciudad. La ubicación en Fase I de costos de las que tienen implantado sistema de costo y el uso de una sola base de distribución para asignar los costos indirectos de fabricación.

\section{IMPORTANCIA DE LOS COSTOS EN LA GESTION ADMINISTRA- TIVA DE LAS EMPRESAS INDUSTRIALES DE CARTAGENA}

Con relación a los precios de venta, el $68.42 \%$ de las empresas que participaron en la encuesta, consideraron que los precios son iguales a los de la competencia, un $15.79 \%$ que son más altos y un $15.79 \%$ que son más bajos. Se deduce entonces, que se está en un sector cuyo mercado es muy competitivo y donde aumentar, el precio de venta de los productos de manera unilateral, sería muy riesgoso, ya que podría ocasionar desplazamiento de los clientes hacia la competencia, al tener ésta, precios más bajos. Por lo tanto, contar con un sistema de costos, que se ajuste a estas exigencias del mercado, sería una fortaleza importante para las empresas de este sector, en la gestión efectiva de sus costos (reducción o ahorros de costos), que conlleven a la consecución de un liderazgo de los mismos, para lograr y mantener la sostenibilidad económica de la empresa en el largo plazo. 
Con relación a los precios por tipo de negocio que son nueve (9) en total, seis consideraron tener precios de ventas iguales a la competencia, donde el porcentaje del negocio de alimentos fue del $60 \%$, el de metalmecánica el $66.57 \%$ y los cuatro restantes fueron el $100 \%$ cada uno. Lo que justifica en cierta forma como se ha comentado, que este sector cuente con un sistema de costos que se ajuste a sus necesidades.

Con relación a los sistemas de costos implantados, se observó que el $52.63 \%$ de las empresas su sistema de costo les permite identificar oportunidades para bajar los costos, mientras que el $47.37 \%$ consideró que no les permite identificar oportunidades. Este resultado demuestra la sub utilización que tienen un gran número (47.37\%) de empresas del sector de su sistema de costos para reducir costos. Circunstancia que las podría colocar en desventaja, por estar en un mercado muy competitivo.

En cuanto si la empresa negocia con base en sus costos, se observó que el $68.42 \%$ si lo hace, el $26.32 \%$ no negocia y el $5.26 \%$ no respondió. De acuerdo con este resultado, se podría deducir que los gerentes conocen en su gran mayoría la importancia que tienen los costos como base para hacer negocios, por lo que es relevante que cuenten con un sistema de costo que les suministre las herramientas necesarias para el acierto en la toma de decisiones.

Finalmente, se evidenció que el $68.42 \%$ de los gerentes no conocen que es el costo basado en actividades y el $31.58 \%$ que si lo conoce. Lo que podría justificar, el hecho de que la gran mayoría de las empresas del sector, estén en Fase I de costos y en algunos casos ni siquiera hayan alcanzado esa etapa. Esta situación, de alguna forma, justifica la realización de esta investigación, en aras de resolver un problema que en la actualidad afecta a un gran número de PYMES del sector industrial de Cartagena.

\section{EXPLICANDO EL PROCESO DE COSTEO}

Consiste en integrar los tres elementos del costo, a través de un diseño de costeo en una hoja de cálculo (Excel), conformado por varias matrices de distribución relacionadas entre sí, que permiten obtener el costeo de cada producto, de acuerdo con sus procesos y actividades relacionadas, utilizando diferentes inductores de costos dependiendo del elemento de que se trate: material directo (MD), mano de obra directa (MOD) y costos indirectos de fabricación (CIF). Para costear los materiales directos se utilizarán los parámetros de consumo por unidad de producto, para el costeo de la mano de obra directa, se utilizará como base de distribución 
las horas hombres de acuerdo con los parámetros de consumo y para los costos indirectos de fabricación, se aplicará el costeo basado en actividades descrito anteriormente, específicamente el planteado por Douglas $\mathrm{T}$. Hicks, por ser una guía específica para pequeñas y medianas empresas. Esta forma de costear, se aplicará a una empresa de lácteos de la ciudad de Cartagena tomada como estudio de caso.

La selección de esta empresa, obedeció a tres aspectos fundamentales a saber: a. por permitir la gerencia el estudio y su aplicación, b. ser esta empresa de alimentos, una de las más numerosas en la ciudad, y c. por tener un proceso que permite ver de manera sencilla y detallada la aplicación del costeo basado en actividades.

Con relación a los materiales directos y la mano de obra directa, no es difícil determinar su inductor, ya que estos se imputan directamente al producto teniendo en cuenta el volumen y las horas hombre de MOD respectivamente. Muy distinto pasa con los costos indirectos de fabricación los cuales tienen diferentes orígenes o causas, por lo que no es objetivo utilizar un solo inductor como base de asignación. En este trabajo se utilizarán diferentes inductores para la asignación de los costos indirectos de fabricación a los procesos, las actividades y a los productos.

\section{Clases De Procesos Y Actividades}

Normalmente los procesos, en una planta de producción son de dos clases: los procesos productivos y los procesos de servicios o de apoyo a la producción.

Por economía, objetividad y facilidad para su uso como herramienta de control y para la toma de decisiones, se procede a agrupar las actividades en centros de actividades, como lo plantea Douglas T. Hicks.

Con relación a los procesos productivos se determinandoscentros deactividades principales: la actividad de transformación y la actividad de empaque. Para los procesos de servicio o de apoyo a planta, se agrupan las siguientes actividades: Calidad, mantenimiento, compras y servicios generales.

\section{Costeo Del Material Directo}

Para determinar el costeo de este elemento, se tienen en cuenta las siguientes variables:

$>$ Precio real de los materiales directos.

$>$ Parámetros de consumos por unidad de producto.

$>$ Producción real del periodo.

> Mermas y desperdicios. 
Estas variables deben actualizarse mensualmente en la matriz de costos en Excel, con el fin de obtener el costo actualizado del material directo, por producto y totalizado por mes, el cual se obtiene multiplicando el precio real de los materiales por la cantidad de acuerdo con los parámetros de consumo para fabricar una unidad de producto.

\section{Costeo De Mano De Obra Directa}

Este se determina teniendo en cuenta el costo real del periodo de los sueldos, las horas extras, el subsidio de transporte, las prestaciones sociales y los aportes, tanto del personal vinculado a la empresa como del personal contratado por bolsa de empleo, y que están relacionados directamente con los procesos productivos.

El costeo por producto, se determina utilizando como base de distribución, las horas hombre de MOD totales del mes por cada proceso, las cuales se calculan teniendo en cuenta la producción real del mes y el requerimiento de horas hombre de MOD para fabricar una unidad de producto, según los parámetros previamente establecidos.

\section{Costos Indirectos De Fabricación}

Su cálculo por producto se realiza teniendo en cuenta los recursos, los procesos, las actividades, y los inductores relacionados con el origen del costo, utilizando como metodología, el costeo basado en actividades (abc). El proceso empieza con la identificación de los recursos y sus costos, la determinación de los CIF de asignación directa a los procesos productivos y a las actividades de apoyo y la distribución de los CIF generales, los cuales necesitan de inductores (drivers fijos y variables), para asignarlos a las actividades productivas y a las actividades de apoyo.

La distribución de los CIF, termina cuando se traslada el costo de las actividades de apoyo a las actividades primarias (recibo, transformación y empaque) y de éstas a los productos. En este sentido el método de distribución de CIF que se utiliza es el escalonado.

En síntesis, éste modelo de costos integra el cálculo de los tres elementos de costo, utilizando como herramienta una hoja de cálculo (Excel), en cuatro etapas.

La primera etapa hace referencia a la identificación o determinación de los parámetros de consumo para fabricar una unidad de producto, en cuanto a materiales directo y tiempos de proceso (horas hombre y horas maquina). La segunda etapa la componen dos planillas, una para acumular los costos de mano de obra directa y la otra para realizar la distribución de la MOD de acuerdo con las horas hombres teóricas. 
La tercera etapa se divide a su vez en tres fases así: la primera consiste en una matriz de distribución, que permite asignar los recursos (CIF directos y los CIF generales) a los procesos productivos y a las actividades de apoyo teniendo en cuenta sus inductores (área, Kw. / hora, Litros), la segunda consiste en distribuir los costos de las actividades de apoyo a las actividades primarias, y la tercera consiste en distribuir las actividades primarias a los productos (objeto final de costo).

En la cuarta etapa se presentan los costos por líneas y por producto, así como la rentabilidad de cada uno.

\section{CONCLUSIONES}

En primera instancia, aunque el objetivo del trabajo realizado, fue conocer en primer lugar en que Fase de costos estaban las empresas del sector, los datos obtenidos hubieran podido ser analizados y tabulados aplicando un método estadístico diferente al utilizado, que brindara la oportunidad de hacer cruce o relación de variables para un análisis más preciso de los resultados. Sin embargo, ese no era el objetivo básico de esta investigación, por tal razón los datos se analizaron y tabularon de forma descriptiva.

Se destaca, la desconfianza por parte de los empresarios y directivos de las empresas para suministrar información contable y de costos, hasta el punto que solo se pudieron aplicar 21 encuestas de 86 empresas disponibles. Sin embargo, muy a pesar de esta dificultad se logró abarcar con las empresas encuestadas todos los tipos de negocios de acuerdo con su participación en la totalidad de las empresas del sector industrial.

Se evidencia, de acuerdo con los resultados de las encuestas, la inexistencia de sistemas de costos en un gran número de PYMES del sector industrial de Cartagena, e incluso, las que dijeron tener implantado un sistema de costos, solo se encuentran en Fase I. Lo que pone de manifiesto el gran riesgo por el que están atravesando este tipo de organizaciones por no contar con un sistema de costos que se ajuste a sus necesidades y responda a los múltiples cambios que hoy presenta el mundo y especialmente el país, en materia económica, social y política.

Cabe resaltar que este trabajo puede ser una guía para que algunas PYMES del sector industrial de esta ciudad, cambien su debilidad actual (no contar con un sistema de costos) en una fortaleza que les permita formular estrategias (liderazgo en costos, reducción de costos, administración basada en actividades o $\mathrm{ABM}$ ), para desarrollar una ventaja competitiva que las lleve a conseguir en el mediano plazo, la sostenibilidad económica que tanto necesitan. 
El hecho de trabajar con tres actividades primarias facilitó la explicación y la aplicación del costeo basado en actividades. Estas actividades en la práctica agrupan una serie de tareas las cuales dependen del tipo de empresa y su proceso de producción. Las otras actividades corresponden a los centros de actividades o actividades de apoyo que también agrupan varias tareas y que por lo general se dan en todas las empresas industriales. Estas actividades son: compras, mantenimiento, calidad y servicios generales.

Finalmente, por todo lo anterior y en aras de que las PYMES del sector industrial de Cartagena, cuenten con una guía para la aplicación del costeo basado en actividades, se realizó un ejercicio tomando como modelo una empresa de productos lácteos, que de ponerlo en práctica las podría colocar muy cerca de la Fase III de costos y con muchas posibilidades de seguir mejorando, para hacer frente a las circunstancias adversas que hoy amenazan a las pequeñas y medianas empresas de la ciudad. En este sentido, se puede afirmar que se ha cumplido con el objetivo general de ésta investigación.

\section{REFERENCIAS BIBLIOGRÁFICAS}

1. Gerts F. Origen y evolución de la contabilidad. México, Editorial Trilla. 1990.

2. Claude G, JR. Historia del pensamiento administrativo. México, Prentice-Hall Hispanoamericana, S.A. 1974.

3. Lorino P. El control de gestión estratégico. Barcelona España. Ediciones Alfaomega Marcombos S.A. 1993.

4. Robert S, Robin C. Coste y Efecto. Ediciones Gestión 2000, S.A., Barcelona. 2003.

5. Del Río C. Costo Integral Conjunto. México, Editorial ECAFSA. 2000.

6. Ramírez Padilla, David Noel. Contabilidad Administrativa. México Editorial Mc Graw-Hill. 7ª Edición. 2005.

7. Hicks D T. El sistema de costos basado en las actividades. Bogota Colombia, Editorial Alfaomega Marcombo, 1998.

\section{BIBLIOGRAFÍA RECOMENDADA}

Goldratt E. El Síndrome del Pajar. Monterrey Nuevo León, México. Ediciones Castillo 1992.

Porter Michael. Competitive Advantage. New York. The Free Press. 1985.

Del Río G. Cristóbal. Introducción al Estudio de la Contabilidad y Control de los Costos Industriales, México, Editorial ECAFSA. 2000. 